\section{BRAZIULIAN JOURNAL}

OF MEDICAL AND BIOLOGICAL RESFARCH

www.bjournal.com.br
ISSN 0100-879X

Volume 44 (7) 606-728 July 2011

BIOMEDICAL SCIENCES

AND

CLINICAL INVESTIGATION

Braz J Med Biol Res, July 2011, Volume 44(7) 682-687

doi: 10.1590/S0100-879X2011007500074

Supraorganized collagen enhances Schwann cell reactivity and organization in vitro

L.G. Maturana, R.G. Zanon, A. Pierucci, B.C. Vidal and A.L.R. Oliveira

The Brazilian Journal of Medical and Biological Research is partially financed by

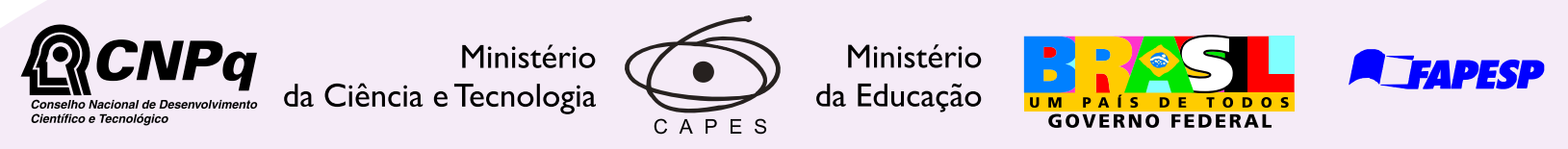

Institutional Sponsors
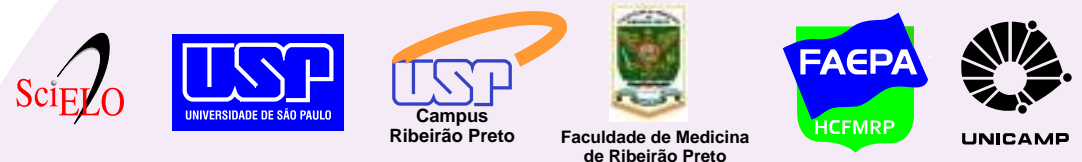

Ф SHIMADZU

GE Healthcare

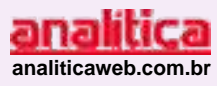

\begin{abstract}
developped by:
\end{abstract}
Thermo
SCIENTIFIC 


\title{
Supraorganized collagen enhances Schwann cell reactivity and organization in vitro
}

\author{
L.G. Maturana, R.G. Zanon, A. Pierucci, B.C. Vidal and A.L.R. Oliveira \\ Laboratório de Regeneração Nervosa, Departamento de Anatomia, \\ Biologia Celular e Fisiologia e Biofísica, Instituto de Biologia, \\ Universidade Estadual de Campinas, Campinas, SP, Brasil
}

\begin{abstract}
We investigated the reactivity and expression of basal lamina collagen by Schwann cells (SCs) cultivated on a supraorganized bovine-derived collagen substrate. SC cultures were obtained from sciatic nerves of neonatal Sprague-Dawley rats and seeded on 24-well culture plates containing collagen substrate. The homogeneity of the cultures was evaluated with an SC marker antibody (anti-S-100). After 1 week, the cultures were fixed and processed for immunocytochemistry by using antibodies against type IV collagen, S-100 and p75NTR (pan neurotrophin receptor) and for scanning electron microscopy (SEM). Positive labeling with antibodies to the cited molecules was observed, indicating that the collagen substrate stimulates SC alignment and adhesion (collagen IV labeling - organized collagen substrate: $706.33 \pm 370.86$, non-organized collagen substrate: $744.00 \pm 262.09$; S-100 labeling - organized collagen: $3809.00 \pm 120.28$, non-organized collagen: $3026.00 \pm 144.63, P<0.05$ ) and reactivity (p75NTR labeling - organized collagen: $2156.33 \pm 561.78$, non-organized collagen: $1424.00 \pm 405.90, P<0.05$; means \pm standard error of the mean in absorbance units). Cell alignment and adhesion to the substrate were confirmed by SEM analysis. The present results indicate that the collagen substrate with an aligned suprastructure, as seen by polarized light microscopy, provides an adequate scaffold for SCs, which in turn may increase the efficiency of the nerve regenerative process after in vivo repair.
\end{abstract}

Key words: S-100; p75NTR; Type IV collagen; Collagen; Cell culture; Supraorganized collagen

\section{Introduction}

Peripheral nerve regeneration is influenced by the chronology and synchronization of a chain of events following injury. The degeneration of the distal stump as well as the sprouting of regenerating fibers are determined by the repair technique, the level of the lesion and the regenerative potential of the axotomized neurons. The simple reconnection of the stumps may not provide all the necessary elements for the most efficient regenerative process, so that the use of different methods has been investigated. There is evidence that the regeneration process may be enhanced if cell grafts are introduced into the lesion microenvironment. In this way, stem cells from different sources and Schwann cells (SCs) combined with different substrates increase the speed, reduce the gap between stumps and the quality of the regeneration process (1-3).

SCs are vital elements during the process of peripheral nerve rearrangement following lesion, the so-called Wallerian degeneration. They support the axonal regrowth from the proximal stump, serving as an efficient interface to the extracellular matrix (ECM) (4). In this respect, they express a number of laminins (4-7) and integrins (e.g., $\alpha 1 \beta 1$, $\alpha 2 \beta 1, \alpha 6 \beta 1)(8)$ and secrete different neurotrophic factors, including nerve growth factor (NGF), brain-derived neurotrophic factor (BDNF) and the glial-derived neurotrophic factor (GDNF) $(9,10)$. Additionally, the SCs organize the so-called bands of Büngner, which provide guidance to the growth cones throughout the distal stump of a lesioned nerve $(4,10,11)$.

The use of purified SCs as a graft inside a tubular prosthesis resulted in a more efficient nerve repair $(1,2,12,13)$. Nevertheless, these effects may be enhanced if the substrate in which the cells are cultivated provides a further functional stimulation and is able to retain the synthesized soluble molecules (14). The possibility of culturing SCs obtained from neonate and adult peripheral nerves also permits a more detailed investigation of the interactions with

Correspondence: A.L.R. Oliveira, Departamento de Anatomia, Biologia Celular e Fisiologia e Biofísica, Instituto de Biologia, UNICAMP, Caixa Postal 6109, 13083-970 Campinas, SP, Brasil. Fax: +55-19-3521-6295. E-mail: alroliv@unicamp.br

Received January 11, 2011. Accepted June 2, 2011. Available online June 17, 2011. Published July 25, 2011. 
the ECM components, as well as the response of such cells when cultured on new substrates, including biomaterials (10-14) and supraorganized ECM-derived molecules.

Overall, collagens are the most abundant ECM elements in the peripheral nerve. They contribute to the structure and function of the nerve, including the connective tissue that is present in the epi-, peri- and endoneural tissue $(4,5)$. Collagen is also present in the basal lamina synthesized by SCs. An interesting feature of collagen fibrils is their ability to become aligned, forming stable supramolecular structures (9). In this way, the fiber architecture itself is a limiting factor for such organization and, although the autoassembly and macromolecular aggregational properties have not been fully investigated, they can be obtained in vitro under controlled conditions.

Naturally oriented proteins are known to positively influence the axonal growth $(14,15)$. They may also be degraded or rearranged more efficiently in the ECM environment. Interestingly, collagen extracted from bovine tendon selfassembles after dialysis. The formation of oriented fibrils can be enhanced by the use of an extrusion technique (16). In contrast, collagen from other sources, such as the rat tail tendon, does not self-organize into helically oriented fibers and chiral objects after extrusion. In this context, there is evidence that supraorganized matrix may be of benefit for cellular recognition and may facilitate the interaction of neuronal and glial cells, increasing axonal guidance.

On the basis of these considerations, the objective of the present study was to investigate the expression of ECM components by SCs when cultured on a naturally aligned collagen substrate. In this way, the expression of type IV collagen, the low affinity receptor for neurotrophins (p75NTR) and $\mathrm{S}-100$ was assessed by immunocytochemistry and the interaction between the SCs and the substrate was studied by scanning electron microscopy (SEM).

\section{Material and Methods}

\section{Collagen extraction and substrate preparation}

Bovine calcanear tendon and rat tail collagen were extracted and separated using a patented technique (\#P.I. 97015709, B.C. Vidal). Briefly, small fragments of defatted and cleaned bovine and rat tendons were immersed in an aqueous solution of $5 \%$ acetic acid containing $0.01 \% \mathrm{HCl}$ and $1 \mathrm{mg}$ pepsin/g tendon at $7-10^{\circ} \mathrm{C}$ for $24 \mathrm{~h}$. The solubilized collagen was filtered and the fibers were reconstituted by adding $\mathrm{NaCl}$ solution to a final concentration of $5 \%$. The fibers obtained were then dialyzed (dialysis membrane with $25 \AA$ porosity, cut off - 12,000-16,000 kDa; Inlab, Brazil) against distilled water in 6-mm diameter tubes at $5^{\circ} \mathrm{C}$, with the outside bath water changed every $24 \mathrm{~h}$ (four times). One and a half liters of water were used for every $200 \mathrm{~g}$ collagen gel.

After lyophilization, the collagen substrate was cut into $5-\mathrm{mm}^{3}$ fragments and sterilized with gamma radiation (15 $k G y)$.

\section{Culture of Schwann cells}

The SC cultures were prepared using sciatic nerves from neonatal Sprague-Dawley rats $(N=10)$. The nerves were dissected out and freed from the epineurium and contaminating tissue. The tissue was then reduced into small fragments and incubated in collagenase and trypsin for $30 \mathrm{~min}$ at $37^{\circ} \mathrm{C}$. The enzymes were inhibited with fetal calf serum and the tissue was triturated, filtered, centrifuged, and resuspended in Dulbecco's modified Eagle's medium with $10 \%$ fetal calf serum. The medium was enriched with forskolin (10 mM, Sigma, USA) and pituitary extract (10 $\mu \mathrm{g} / \mathrm{mL}$; Sigma). The cells thus obtained were then seeded onto 24-well culture plates containing the polymer membranes. After $24 \mathrm{~h}$ of culturing, a further cell purification was performed by adding $10 \mathrm{nM}$ cytosine arabinoside for $48 \mathrm{~h}$. The purity of the cultures was confirmed with the anti-S-100 antibody (Dako, Denmark). The cultures, once purified, were maintained for 1 week on the biopolymers and the medium was changed every second day. All experiments were performed in triplicate and were approved by the Institutional Committee for Ethics in Animal Research, Universidade Estadual de Campinas (UNICAMP, protocol No. 1360-1).

\section{Immunocytochemistry}

After 1 week of culture, the cells were fixed in $4 \%$ paraformaldehyde (Merck, Germany) for $10 \mathrm{~min}$ and washed twice in $0.1 \mathrm{M}$ phosphate buffer (PB), $\mathrm{pH} 7.4$, for $5 \mathrm{~min}$. Preincubation with $1 \%$ bovine albumin for 45 min was followed by overnight incubation at $4^{\circ} \mathrm{C}$ with primary antisera (anti-S-100, anti-p75NTR, and anti-type IV collagen; Santa Cruz Biotechnology Inc., USA). After three rinses in PB, the cultures were incubated for $45 \mathrm{~min}$ at room temperature with the conjugated donkey anti-goat or anti-rabbit secondary antibodies Cy-2 or Cy-3 (Jackson Immunoresearch Laboratories Inc., USA) according to the primary antibodies. After rinsing in $\mathrm{PB}$, the preparations were mounted in a mixture of glycerol/PB (3:1) and examined with a Nikon inverted microscope equipped with epifluorescence and appropriate filter combinations for the fluorophores used. The immunofluorescence was quantified with a plate reader (Synergy 2 Multi-Mode Microplate Reader, Biotek, USA). For this purpose, SCs were cultured on bovine or rat collagen (non-organized substrate, control) in 96-well special optic plates designed for fluorescence assays (07-200-730, Fisher Scientific, USA). Blank as well as collagen only wells were used as negative controls and the respective reading values were subtracted from the experimental results. All readings were performed in quadruplicate $(\mathrm{N}=4$ for each antibody analyzed). The readings were obtained using the fluorescence detection method and the endpoint reading option of the GEN5 ${ }^{\mathrm{TM}}$ software (Microplate data collection and analysis software, version 1.10, Biotek, USA). Also, the bottom optic position was selected and the sensitivity of the readings was set to maximum. The results were subjected 
to ANOVA and differences between groups were analyzed by the Student $t$-test, with the level of significance set at $\mathrm{P}<0.05$. Data are reported as means \pm standard error of the mean.

\section{Preparation for scanning electron microscopy}

The cultures were fixed in Karnovsky's fixative ( $2 \%$ glutaraldehyde and $1 \%$ paraformaldehyde in $0.1 \mathrm{M}$ cacodylate buffer, pH 7.4; Fluka, Germany) for 15 min. A secondary fixation with $1 \%$ osmium tetroxide was performed, followed by washing with buffer and dehydration in ethanol $(30,50$, 70,90 , and $100 \%$, 15 min per step). The specimens were then taken to the critical point dryer (Balzers, CTD030, Liechtenstein) and coated with gold for $120 \mathrm{~s}$ at $20 \mathrm{~mA}$ using a sputter coater, resulting in an approximate coating thickness of $40 \mathrm{~nm}$. The specimens were then viewed under an SEM (Jeol, JXA 840A, Japan) operated at $10 \mathrm{kV}$.

\section{Results}

The collagen substrate was analyzed by polarized light microscopy and SEM. The general organization of the fibrils is illustrated in Figure 1A. A parallel pattern could be seen throughout different focus planes, indicating the suprastructural organization of the substrate. This was confirmed by SEM, which also provided evidence of the presence of multiple layers of fibrils (Figure 1B). Combined with the light microscopy observations, it was possible to conclude that such layers were arranged in a three-dimensional helical fashion.

SEM analysis of SCs reinforced the integration with the organized collagen substrate (Figure 1C). Also, the cells tended to present a longitudinal organization, resembling the formation of the bands of Büngner in vivo.

Examining the SC culture, under phase contrast, it was possible to observe that SCs displayed their characteristic bipolar morphology with thin cell processes. The immunolabeling against S-100 revealed that the cultures were homogeneous, as seen in Figure 1D. Interestingly, SCs cultured on collagen presented longer cell projections, which were penetrating the substrate, indicating a three-dimensional organization of the cells.

Regarding ECM expression, observed by immunocytochemistry, it was possible to identify immunolabeling against type IV collagen both in the control and substratecultured cells (Figure 2J and L). Nevertheless, quantification did not show statistical differences between the cultures seeded on bovine- or rat-derived collagen.

The analysis of S-100 and p75NTR immunoreactivity is presented in Figure 2B, D,
$\mathrm{F}$, and $\mathrm{H}$ and indicates a better alignment of cells cultured on the bovine substrate. Also, the quantitative data revealed a statistically significant increase of immunoreactivity when SCs were cultured on supraorganized bovine-derived collagen (Figure 2, bottom).

\section{Discussion}

The repair of a transected peripheral nerve in which the stumps are separated by a distance is a challenging medical problem. Although the use of autografts is the option of choice, in many instances the size of the gap and the caliber of the nerve require a significant amount of nerve grafts, compromising sensory functions at the donor site. Thus, the possibility of using artificial implants to bridge a nerve defect has been pursued for some time. In particular, the tubulization technique has proved to be an alternative to nerve grafting over small gaps. Also, the use of ECM components within the tubular prosthesis made it possible to increase the gap between the stumps and still obtain a satisfactory guided axonal regeneration.

In order to further enhance the regeneration process, the use of three-dimensional scaffolds has become necessary. The three-dimensional organization of the implant may facilitate cell migration and tissue neoformation leading to a faster repair of the lesion. Collagen-based fibrous scaffolds were produced by Yow et al. (17) and provided an efficient substrate for the spatial organization and proliferation of

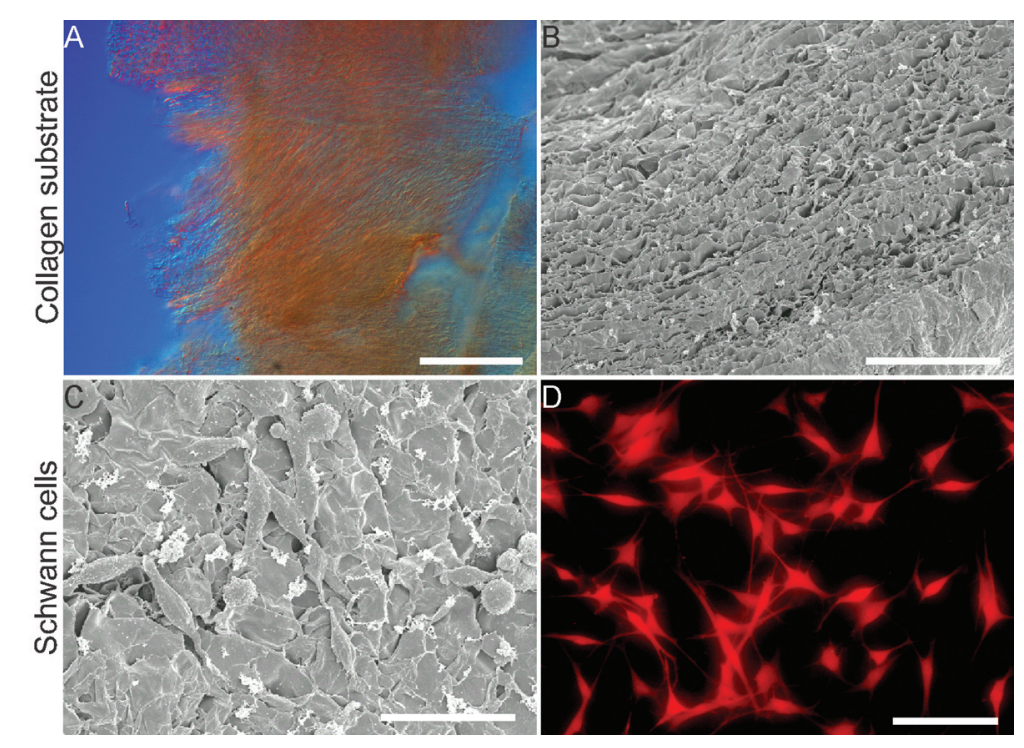

Figure 1. $A$, Representative samples of supraorganized collagen depicting the parallel arrangement of the fibrils ( $b a r=100 \mu \mathrm{m})$. $B$, Collagen substrate observed by scanning electron microscopy. Note the multilayer organization as well as the porosity of the material $(\mathrm{bar}=100 \mu \mathrm{m})$. $C$, Schwann cells cultured on the collagen substrate. Observe the adhesion shown by the cell projections over the surface of the material $(\mathrm{bar}=50 \mu \mathrm{m})$. $D$, Representative example of a purified Schwann cell culture immunolabeled against S-100 (bar $=10 \mu \mathrm{m})$. 

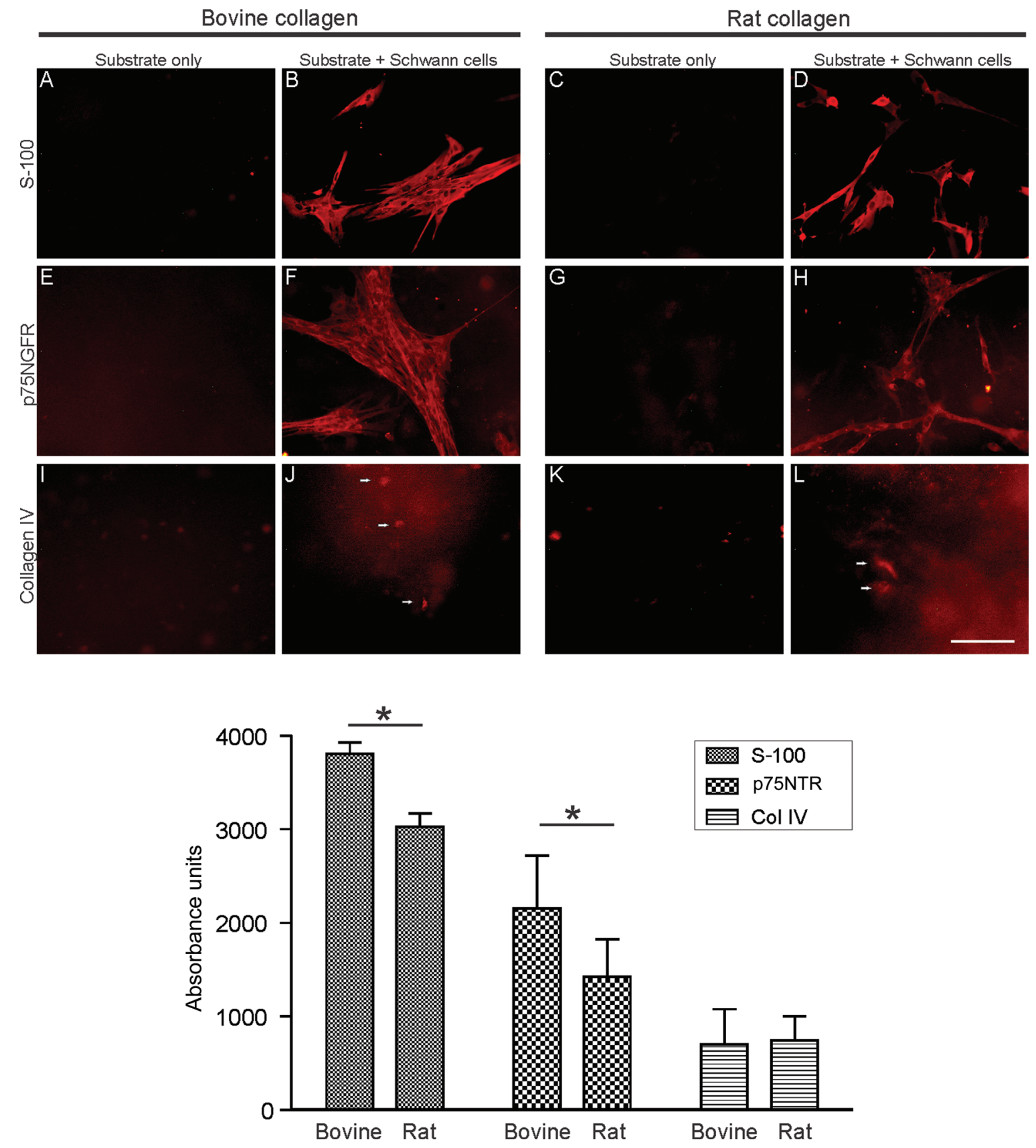

Figure 2. Comparison between Schwann cell cultures performed on supraorganized (bovine collagen) and on non-organized collagen (rat collagen). $A$ and $C$, Substrate only (control) immunolabeled against $\mathrm{S}-100$. $B$ and $D$, Schwann cells cultured on bovine and rat collagen, respectively, and immunolabeled against S-100. Note the better alignment of SCs on the organized collagen. $E$ and $G$, Substrate only (control) immunolabeled against p75NTR (p75 pan neurotrophin receptor). $F$ and $H$, Schwann cells cultured on bovine and rat collagen, respectively, and immunolabeled against p75NTR. Note the better alignment and increased number of SCs on the organized collagen. I and $K$, Substrate only (control) immunolabeled against type IV collagen. $J$ and $L$, Schwann cells cultured on bovine and rat collagen, respectively, and immunolabeled against type IV collagen. No differences were observed between the different substrates. Labeled cells are indicated by arrows. Bar $=100$ $\mu \mathrm{m}$. The bottom panel contains the quantitative data for the immunolabeling against S-100 (B and D), p75NTR $(F$ and $H)$ and type IV collagen (Col IV, J and L). Data are reported as means \pm standard error of the mean. ${ }^{*} P<0.05$ (one-way ANOVA). 
mesenchymal stem cells. These cells have been shown to enhance peripheral nerve regeneration after tubulization repair (3). Also, the concept that molecular alignment of collagen facilitates axonal growth and nerve regeneration has been proposed by Dubey et al. (15), Ceballos et al. (18), and Oliveira et al. (16).

Dubey et al. (15) and Ceballos et al. (18) used a magnetic field to force the arrangement of collagen fibrils, so that a birefringent gel was obtained. In the present study, the molecular supraorganization was obtained naturally due to the maintenance of the self-assembly properties of the collagen after acid extraction and dialysis. This is particularly beneficial because it results in a stable suprastructured substrate, as seen by SEM analysis.

The efficiency of such naturally supraorganized collagen substrate was assessed by analyzing SC immunoreactivity against S-100, p75NTR and type IV collagen. These three molecules are important elements regarding SC response to a nerve injury. In this way, the increased levels of the low-affinity receptor for neurotrophins (p75NTR) observed in supraorganized collagen cultures indicate an improved reactivity state that may further stimulate in vivo regeneration. The present results also demonstrate that SCs populate the three-dimensional scaffold, as shown by the presence of S-100-positive cells on different focal planes.

\section{References}

1. Abernethy DA, Thomas PK, Rud A, King RH. Mutual attraction between emigrant cells from transected denervated nerve. J Anat 1994; 184 (Part 2): 239-249.

2. Madison RD, Archibald SJ. Point sources of Schwann cells result in growth into a nerve entubulation repair site in the absence of axons: effects of freeze-thawing. Exp Neurol 1994; 128: 266-275.

3. Pereira Lopes FR, Camargo de Moura Campos L, Dias Correa J Jr, Balduino A, Lora S, Langone F, et al. Bone marrow stromal cells and resorbable collagen guidance tubes enhance sciatic nerve regeneration in mice. Exp Neurol 2006; 198: 457-468.

4. Ide C. Peripheral nerve regeneration. Neurosci Res 1996; 25: 101-121.

5. Sunderland $\mathrm{S}$. The anatomy and physiology of nerve injury. Muscle Nerve 1990; 13: 771-784.

6. Timpl R, Brown JC. Supramolecular assembly of basement membranes. Bioessays 1996; 18: 123-132.

7. Badylak SF, Freytes DO, Gilbert TW. Extracellular matrix as a biological scaffold material: Structure and function. Acta Biomater 2009; 5: 1-13.

8. Milner R, Wilby M, Nishimura S, Boylen K, Edwards G, Fawcett J, et al. Division of labor of Schwann cell integrins during migration on peripheral nerve extracellular matrix ligands. Dev Biol 1997; 185: 215-228.

9. Zochodne DW. The microenvironment of injured and regenerating peripheral nerves. Muscle Nerve Supp/ 2000; 9: S33-S38.

10. Schmidt CE, Leach JB. Neural tissue engineering: strategies
An important point to be emphasized is the fact that collagen is a natural component of the ECM of the nerve, so that its integration in such microenvironment is possibly better than that of artificial biopolymers. Also, the degradation and reabsorption should be faster and less susceptible to inflammatory reactions in the case of collagen. Another important fact to be considered is that the better adhesion and integration to the substrate results in synthesis of neurotrophic factors, as already demonstrated in SC cultures (19) and supported here with regard to the immunoreactivity against p75NTR. These conditions also induce the formation of bands of Büngner, which are fundamental for the nerve regeneration process (14).

On the basis of the present data, we believe that the use of supraorganized collagen substrates enhances the response of SCs in vitro. The scaffold plus SCs produced in the present study may increase the peripheral nerve regeneration if used as an implant combined with a tubular prosthesis.

\section{Acknowledgments}

A.L.R. Oliveira is the recipient of a fellowship from $\mathrm{CNPq}$ (\#300789/2009-2). for repair and regeneration. Annu Rev Biomed Eng 2003; 5: 293-347.

11. Stoll G, Jander S, Myers RR. Degeneration and regeneration of the peripheral nervous system: from Augustus Waller's observations to neuroinflammation. J Peripher Nerv Syst 2002; 7: 13-27.

12. Evans GR, Brandt K, Katz S, Chauvin P, Otto L, Bogle M, et al. Bioactive poly(L-lactic acid) conduits seeded with Schwann cells for peripheral nerve regeneration. Biomaterials 2002; 23: 841-848.

13. Schlosshauer B, Muller E, Schroder B, Planck H, Muller HW. Rat Schwann cells in bioresorbable nerve guides to promote and accelerate axonal regeneration. Brain Res 2003; 963: 321-326.

14. Ribeiro-Resende VT, Koenig B, Nichterwitz S, Oberhoffner $\mathrm{S}$, Schlosshauer B. Strategies for inducing the formation of bands of Büngner in peripheral nerve regeneration. Biomaterials 2009; 30: 5251-5259.

15. Dubey N, Letourneau PC, Tranquillo RT. Guided neurite elongation and Schwann cell invasion into magnetically aligned collagen in simulated peripheral nerve regeneration. Exp Neurol 1999; 158: 338-350.

16. Oliveira ALR, Vidal BC, Langone F. Naturally supraorganized collagen increases axonal regeneration after tubulization repair. Braz J Morphol Sci 2005; 22: 143-148.

17. Yow SZ, Quek CH, Yim EK, Lim CT, Leong KW. Collagenbased fibrous scaffold for spatial organization of encapsulated and seeded human mesenchymal stem cells. Biomaterials 2009; 30: 1133-1142. 
18. Ceballos D, Navarro X, Dubey N, Wendelschafer-Crabb G, Kennedy WR, Tranquillo RT. Magnetically aligned collagen gel filling a collagen nerve guide improves peripheral nerve regeneration. Exp Neurol 1999; 158: 290-300.
19. Pierucci A, Duek EA, de Oliveira AL. Expression of basal lamina components by Schwann cells cultured on poly(lactic acid) (PLLA) and poly(caprolactone) (PCL) membranes. $J$ Mater Sci Mater Med 2009; 20: 489-495. 